\title{
Variación de parámetros de calidad del grano y aceite de girasol almacenado a diferentes humedades relativas.
}

\author{
Por A. Motta y G. M. Eguiazu
}

INCABIE, Facultad de Ciencias Agrarias / CONICET / UNR - Berutti 1886 - 2000 Rosario - ARGENTINA

\section{RESUMEN}

Variación de parámetros de calidad del grano y aceite de girasol almacenado a diferentes humedades relativas.

Muestras comerciales de girasol fueron sometidas a distintas humedades relativas ambiente (75\%, 80\%, $84 \%, 92 \%$ y $98 \%$ ) durante dos meses. Además de la rehidratación cinética, en las muestras testigos y tratadas, se midieron los siguientes parámetros: humedad, riqueza grasa, poder germinativo, índice de acidez, indice de iodo y composición en ácidos grasos.

La composición en ácidos grasos, el índice de iodo y la riqueza grasa no sufrieron variaciones significativas.

Los parámetros más sensibles que pusieron en evidencia el deterioro fueron el índice de acidez y el poder germinativo.

PALABRAS-CLAVE: Almacenamiento - Calidad (parámetros) - Girasol - Humedad relativa.

\section{SUMMARY}

Variation of the quality parameters of sunflower grain and oil ( $H$. annus) stored at different relative environmental humidities

Commercial samples of sunflower were exposed to different relative humidities $(75 \%, 80 \%, 84 \%, 92 \%$ and $98 \%)$ for two months. In addition to the kinetic rehydratation in standard and treated samples, the following parameters were measured: humidity, fat content, germinative power, acidity index, iodine number and fatty acids composition.

Fatty acids composition, iodine number and fat content, did not change significantly. Only the acidity index and the germinative power were an index of the damage.

KEY-WORDS: Quality (parameters) - Relative humidity Storage - Sunflower.

\section{INTRODUCCION}

La rehidratación lenta de los granos de girasol, en diversas humedades relativas ambiente y la permanencia de los mismos en equilibrio durante un tiempo determinado, permite estudiar en forma controlada el fenómeno de deterioro espontáneo por enmohecimiento y la posible aparición de micotoxinas (1) (7) (17).
Este fenómeno cinético, ejerce una influencia sobre parámetros comerciales conocidos: la humedad y el índice de acidez, a la vez que se ve influido por la constitución anatómica del grano y el contenido de materia grasa. El deterioro producido luego de la rehidratación cinética a elevadas humedades relativas ambiente, comienza mucho antes de que la masa fúngica y la producción de micotoxinas llegue a niveles tales que puedan ser medidos (3) (4) (10).

El objeto de este trabajo es el de investigar la posible influencia que puede existir entre distintas condiciones de rehidratación cinética, sobre los parámetros comerciales conocidos y además de los ya mencionados, fundamentalmente sobre aquellos que tienen relación directa con la calidad del aceite extraido, a saber la composición de los ácidos grasos y el Indice de lodo.

Asimismo, la posibilidad de encontrar algún indicador químico sería de aplicación para detectar o servir como señal de alarma de aquellas condiciones de almacenamiento que pudieran originar el deterioro o el inicio del deterioro biológico de los granos y poder así revertir el proceso cuando aún se halla a tiempo, principalmente en lo referente a las micotoxinas, fundamentalmente la aflatoxina, que al constituir un deterioro de la calidad biológica y ecotoxicológica del grano, la dificultad de su eliminación una vez formadas, hace necesario se investiguen las vías de profilaxis a su aparición, pese a que, como se ha indicado (14) (15), la medición de la Humedad Relativa Ambiente, contribuye en manera principal a este objetivo (9).

\section{MATERIALES Y MÉTODOS}

De las muestras de girasol cosechado que ingresaron a la Cámara Arbitral de Cereales de la 
Bolsa de Comercio de Rosario, muestras de partidas que serán destinadas a la industrialización o almacenamiento, se tomaron al azar, cinco muestras. A cada una de ellas se la subdividió en seis submuestras: una como testigo y cinco para los ensayos. En cinco cubas de vidrio de cierre hermético, mediante el empleo de soluciones saturadas de sales (2), se obtuvieron humedades relativas de $75 \%, 80 \%, 84 \%, 92 \%$ y $98 \%$ y se colocaron una submuestra de cada una de las muestras de girasol.

Las cubas se ubicaron en una cámara de clima controlado, permaneciendo durante todo el período de almacenaje a $25^{\circ} \mathrm{C}$, más o menos $0,2^{\circ} \mathrm{C}(10)$.

El tiempo de almacenamiento fue de 60 días a fin de que, logrando el equilibrio higroscópico ya a los 30 días, poder lograr en el tiempo restante el deterioro o la continuación del deterioro biológico de las muestras.

Al comienzo y fin del ensayo, se midió el peso de cada submuestra. Periodicamente se observaban los ensayos para detectar la aparición de micelio.

A cada testigo y submuestras luego de la experiencia, se les analizó:

a) Contenido de Humedad.

b) Contenido de Materia Grasa.

ambos, según las técnicas detalladas por la Junta Nacional de Granos en la Resolución $N$ ․․ 23.525 del 29/10/82.

c) Acidez de la Materia Grasa: Norma IRAM 5512-N10 (11)

d) Ácido Grasos (del aceite extraido según b) según Norma IRAM 5651 y Norma IRAM 5650 (12).

e) Indice de lodo: por empleo de los valores de ácidos grasos y aplicación de constantes.

f) Poder Germinativo: Según Normas ISTA.

\section{RESULTADOS Y DISCUSION}

En la Tabla anexa se detallan los valores analíticos y de ganancia de peso por absorción de agua, indicada esta última en la columna "2". En la columna " 3 " se indican los valores de Humedad Real, la obtenida por el método analítico y la Humedad Teórica, obtenida por la ganancia de agua por absorción de la atmósfera circundante y el contenido de humedad inicial.
La diferencia entre los valores de humedades teórica y real, sobre todo en los tratamientos a elevadas Humedades Relativas, se explicaría por la pérdida de masa del grano por la actividad biológica con la producción de $\mathrm{CO}_{2}$, no apreciándose por este motivo, el aumento de peso por absorción de humedad, siendo por ello, el valor de Humedad teórica inferior al real, como puede observarse sobre todo en los tratamientos a $92 \%$ y $98 \%$ de las muestras "III", "IV" y "V". Otro factor que contribuye a la diferencia entre los valores de humedad teórica y real, es el agua generada por el proceso de respiración, sobre todo en las muestras con elevado contenido de materia grasa, las cuales al cabo de los 60 días se encontraban con un importante grado de deterioro biológico (caracterizado por el grado de enmohecimiento de las muestras). Este agua generada por la respiración es medida por el método analítico, no así por el cálculo en base a la ganancia de peso.

Se observa también, que en muestras con elevado contenido de materia grasa, la respiración aportaría mayor cantidad de agua que en muestras con menor contenido de materia grasa.

La actividad biológica, explica también el porque los valores de humedad real en las submuestras, sobre todo en los tratamientos a elevadas Humedades Relativas y de las muestras con elevado contenido de materia grasa, superan ampliamente los valores de contenido de humedad que, para iguales valores de humedad relativa, alcanzan en el equilibrio muestras con igual contenido de materia grasa (10). En la columna "4" se indican los valores de Materia Grasa, parámetro que, en valor absoluto, no sufriera grandes modificaciones.

En la columna "5", se indica el valor de Indice de Acidez. Este sí pone en evidencia cambios significativos, sobre todo a elevadas humedades relativas y en aquellas submuestras correspondientes a muestras con elevado contenido de materia grasa, aspecto ya observado en trabajos anteriores (2) (7) (8), lo cual indica la mayor suceptibilidad de deterioro de las mismas.

El Indice de Acidez da una idea de la cantidad de ácidos grasos que fueron escindidos en el proceso de deterioro.

En la columna "6", se indican los valores de Indice de lodo. Este parámetro tampoco sufrió cambios significativos.

En la columna "7", se indica el valor de Poder Germinativo de los testigos y submuestras sometidas a los tratamientos. Como era de esperar, el incremento del deterioro es mayor, cuanto mayor 


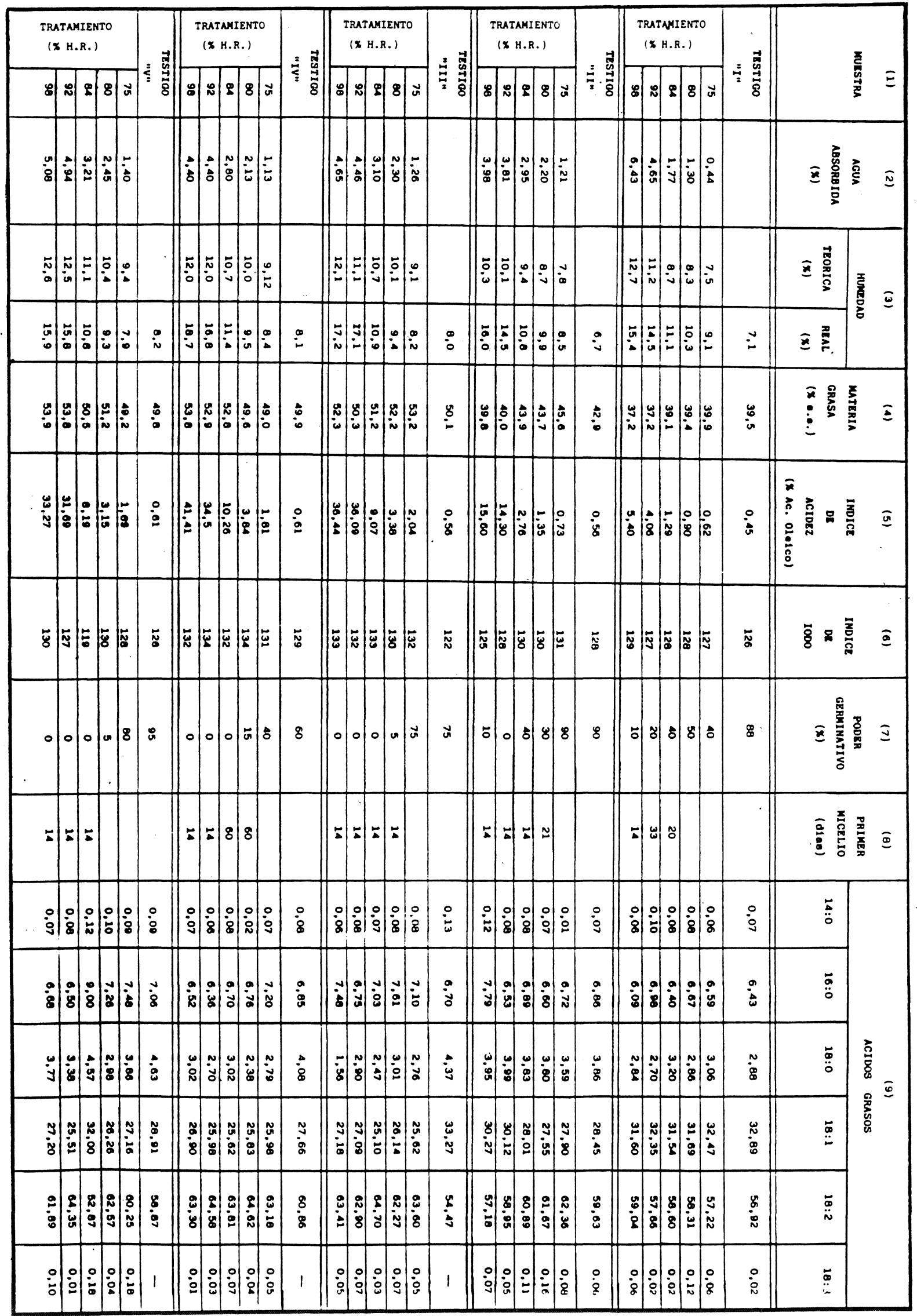


es la Humedad Relativa a la cual fueron sometidas la submuestras. No obstante, las submuestras correspondientes al Testigo "l", con bajo contenido de materia grasa, aún evidencia capacidad de germinación en el tratamiento correspondiente a $98 \%$ de H. R. luego de los 60 días de almacenamiento, mientras que, las submuestras correspondientes a los Testigos "III", "IV" y "V", con mayor contenido de materia grasa, han perdido toda capacidad de germinación ya a partir del tratamiento a $84 \%$ de Humedad relativa.

Las submuestras correspondientes al Testigo "Il", presentan un comportamiento similar a las correspondientes al Testigo "I". La disparidad entre los valores correspondientes a los tratamientos a $92 \%$ y $98 \%$ de H. R., $0 \%$ y $10 \%$ respectivamente, de Poder Germinativo, se explica por la presencia de plántulas que pese a haber germinado, por ser "anormales" no son incluidas, su valor, en el valor de Poder Germinativo.

En la columna "8" se indica el tiempo en la aparición del Primer Micelio (5) (6). Para algunos tratamientos, el tiempo de aparición es menor que el encontrado en un trabajo anterior, para iguales valores de humedad relativa, ello se explicaría por la historia previa de dichas muestras, las que pudieron haber sufrido un enmohecimiento previo a la cosecha, el cual, aunque leve, determina una mayor cantidad de esporas y micelio latente a diferencia de aquellas muestras que al madurar en condiciones ambientales favorables, no sufren ningún tipo de deterioro, llegando a cosecha en óptimo estado de sanidad. Esto se observa también en el tratamiento a $84 \%$ de H. R. correspondiente al testigo "I". En dicha submuestra, la aparición de micelio es anterior que para la submuestra correspondiente al tratamiento a $92 \%$ de H. R. y se explicaría por la presencia, en la submuestra correspondiente al tratamiento a $84 \%$ de H. R., de una partícula, grano o materia extraña, con ataque fúngico previo.

En la columna "9" se indican los valores de los Acidos Grasos: Mirístico (14:0), Palmítico (16:0), Esteárico (18:0), Oléico (18:1), Linoléico (18:2) y Linolénico (18:3). Dichos valores, tampoco muestran una variación significativa, aún en aquellas submuestras sometidas a tratamientos a elevadas humedades relativas. Este aspecto no ocurrió en un trabajo realizado sobre soja donde aumentó el $\%$ de Acido Oléico y disminuyó el \% de Acido Linolénico (16).

\section{CONCLUSIONES} que:

Por lo observado podemos concluir diciendo

1. Respecto a la calidad industrial (13) (18), es interesante constatar que la calidad del aceite no ha sufrido grandes cambios, pero sí es de esperar una pérdida por neutralización durante el proceso industrial, por los altos Indices de Acidez.

2. El mejor valor para medir el deterioro de la calidad comercial es el Indice de Acidez, no así la composición de los ácidos grasos y el Indice de lodo.

3. Debe pensarse que el deterioro de la calidad biológica y ecotoxicológica, fundamentalmente por la aparición de micotoxinas, puede ocurrir en estas muestras, por el nivel del desarrollo fúngico ocurrido principalmente a elevadas Humedades Relativas y por lo observado en trabajos anteriores que emplearon condiciones similares de almacenamiento, a pesar que la composición de los ácidos grasos del aceite de dichas muestras no sufrieran modificaciones.

4. Los granos con mayor contenido de materia grasa, serían más sensibles al deterioro biológico, apoyando lo ya observado en un trabajo anterior donde se indicara y postulara como "hipótesis antropoecológica" que al apartarse el proceso de selección de los Helianthus primigenios y buscar variedades o hibridos de elevado contenido de aceite, los mismos son más suceptibles o sensibles al deterioro biológico, no sólo de la calidad comercial e industrial, sino y más importante de la calidad biológica y ecotoxicológica (10).

\section{AGRADECIMIENTOS}

- A la Cámara Arbitral de Cereales de la Bolsa de Comercio de Rosario por su colaboración en la realización de los análisis.

- A la Ingeniería Química Susana Abrate, nuestro especial reconocimiento por su valiosa colaboración prestada en la determinación por cromatografía gaseosa, de la composición de ácidos grasos de las muestras de aceite.

- Al Ingeniero Carlos A. Perigo, por la determinación de los valores de poder germinativo. 


\section{BIBLIOGRAFIA}

1. Eguiazu, G. M.- "Micotoxinas en maiz: resultados analíticos del aislamiento de Aflatoxinas y su agente causal en maíz de la zona de influencia de Rosario".- Bolsa de Comercio de Rosario.- 69 págs., 1978.

2. Eguiazu, G. M.- "Bildungsbedingungen von Aflatoxinen in Sonnenblumen".- Tesis doctoral (Dr. Scienciarum Agrarium) Universität Stuttgart-Hohenheim, 1983.

3. Eguiazu, G. M.- "Comportamiento de almacenaje de girasol. I. Comportamiento de absorción de la humedad atmosférica del aquenio, pericarpio y semilla del grano de girasol".- Grasas y Aceites 35 (1984) 246-250.

4. Eguiazu, G. M. y Grünewald, T.- "Comportamiento de almacenaje de girasol. II. Cinética de la absorción para muestras de variado origen".- Grasas y Aceites 35 (1984) 320 324.

5. Eguiazu, G. M.- "Comportamiento de almacenaje de girasol. III. Aparición del primer micelio visible como primer síntoma de deterioro, en función de la humedad crítica de la muestra, la humedad relativa ambiente y el tiempo de almacenaje".- Grasas y Aceites 35 (1984) 325-329.

6. Eguiazu, G. M. y Frank, H. K.- "Counts of Fungal Propagules and acid number in sunflower seeds".- Fette Seifen Anstrichmittel 86 (1984) 16-18.

7. Eguiazu, G. M.- "Comportamiento de almacenaje de girasol. V. Aparición espontánea de Aflatoxina y Esterigmatosistina en girasol almacenado bajo aire y $90 \%$ de $\mathrm{CO}_{2}{ }^{\prime}$.Grasas y Aceites 37 (1986) 25-28.

8. Eguiazu, G. M.- "Comportamiento de almacenaje de girasol. IV. Efecto de alto porcentaje de anhídrido carbónico en la atmósfera del almacenaje sobre la conservabilidad del girasol" ${ }^{n}$ - Grasas y Aceites 35 (1984) 378-383.

9. Eguiazu, G. M. y Motta, A.- "The relative atmospheric humidity of the intergranular air as a parameter of the biological damage in stored sunflower".- Fette Seifen Anstrichmittel 88 (1986) 168-173.
10. Eguiazu, G. M. y Motta, A.- "Relación entre contenido de agua y materia grasa en muestras comerciales de girasol para una humedad relativa ambiente del aire intergranular del 75\%".- Grasas y Aceites 37 (1986) 307-312.

11. IRAM (1955) "Determinación de la acidez de la materia grasa" NI 5512-N10.

12. IRAM (1976) "Norma para aceites vegetales y animales" Determinación por cromatografía en fase gaseosa de ésteres metílicos de ácidos grasos NI 5651 y preparación de ésteres metílicos NI 5650 .

13. Kirschenbauer.- "Grasas y Aceites, Química y Tecnología".Ed. Continental.- 310 págs., 1964.

14. Motta, A.- "Profilaxis del deterioro fúngico y formación de micotoxinas en base a la medición de la humedad relativa ambiente del aire intergranular" - Bolsa de Comercio de Rosario.- 29 págs., 1987.

15. Northolt, M. D. y Heuvelman, C. J.- "El ensayo de licuación de sales cristalinas, método sencillo para medir la actividad de agua de los alimentos ${ }^{n}$.- Manual de la OCDE.Madrid, España, 1982.

16. Patil, K. B. et al.- "Effect of fungi on the lipid composition of soybean during storage at different levels of humidity".Fette Seifen Anstrichmittel 88 (1986) 18-19.

17. Reis, J.- "Mykotoxine in Lebensmitteln".- Gustav Fischer Verlag.- 549 págs., 1981.

18. Zarembo, G. V. and Matsuk, P.- "Losses of oil during the manufacturing process. Causes and remedies".- Maslozhir. Prom. (9) (1979) 1-6.

(Recibido: Diciembre 1990) 